\title{
Identification of Probability of thermally induced Transient Vibration in CentrifugalCompressor
}

\author{
Mantosh Bhattacharya, \\ Rotating Equipment Specialist UAE
}

\begin{abstract}
It has been observed that a phenomenon known as "transient vibration "sometimes occurs in centrifugal compressorswith between bearing configuration during start- up just after a short shutdown. The phenomenon iscaused by a thermal induced bow which is similar to rotor bow occurring in large steam turbines during start-up.The occurrence of this phenomenon depends on certain operating conditions and loop configurations of centrifugal compressors. The temporary rotor bow may cause rotor -stator rubbing due to vibration at high amplitude during restart of compressor inthe hotcondition.This write up endeavors to provide a guideline about when a method to assess sensitivity to rotor bow during testing of compressors at manufacturer's test bed. Without identifying the potential severity of this issue it is very cumbersometo change design or implement protective logics in system.
\end{abstract}

Keywords: Phase, slow-roll, stiffness ratio, logic solver

\section{Introduction}

Centrifugal compressors are mission critical machinery and stoppage of such machines in an important process loop leads to production downtime and loss of inventory.

Machinery and associated process loopprotection is carried out by attenuating alarm and trips taking into view to minimize machinery outage time.Apartfrom onerous alarm/ trip annunciations, there are some benign high vibration signals, signal error or controller error, coupling guard noiseissues, spurious trips etc. which are mended quickly and compressor is started again after a short shutdown.

High radial vibration trips/ failures were encountered while starting up centrifugal compressorsat field after a short shutdown (known as hotre-start). The vibrationwas highest at first resonant speed. Spurious alarm / trip annunciation indicating dry gas seal failure were also experienced.

The high 1Xvibration normallyis a symptom of imbalance which iseither caused by high eccentric mass center of rotor or skewed impeller or shaft with a residual bow. The balancing record of rotor and coupling,imbalance response test results of compressors in vendor shop, alignment data showed all values were well within acceptance criteria. In some of cases, where the rotors accelerated throughthe HH vibration trip value with very low margin,the vibration amplitude came back to normal at its operating speed after some time. The reason was finally concluded as high transient eccentricity caused by rotor thermal transient bow phenomenon.

Unlike permanent bow due to gravity sag of rotor,it was identified that this type of bow occurs due to uneven temperature distribution circumferentially around the rotor i.e. differential heating / cooling of rotor. As the compressor is shutdown, the rotor starts dissipating heat as following manner -

The convective heat transfer from lower part of rotor is more than with respect to upper part(a case of differentialcooling). See the convection current in blue in fig 1.The higher temperature pole will elongate in the axial direction more than the other pole and, as a result, the rotor will bow in resultant direction.

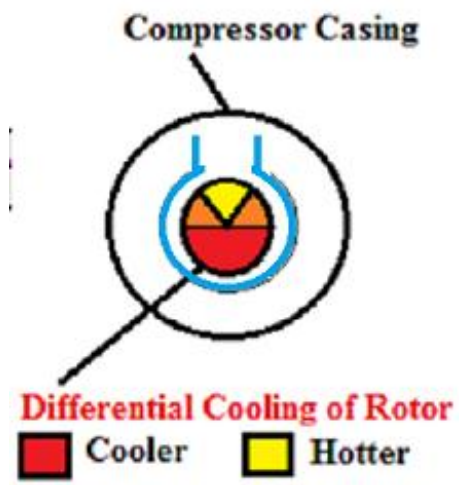

Fig 1- Transient thermal instability phenomena in rotor 
It is worth noticing that rotor static deflection also plays a significant role in setting up the direction of rotor bow as shown in figure.

The mathematical treatment of this phenomena is explained in detail by Baldassarre et al [1]

Differential heating of rotor can happen due to hot seal gas supply by seal gas heaters if inadvertently kept on during long pressurized standby condition of compressor.

It is interesting to know that all compressors do not exhibit such phenomenon although it occurs in all compressor rotors the reasons shall be explained in later portion of this paper. There are many governing factors involved like rotor construction and rotor stiffness, settle out pressure and temperature construction and process condition just before stoppage and time taken to restart. Rotor settle pressure plays a role in convective heat transfer as the phenomena implicitly dependent on pressure and temperature of surroundingarea and thermal dilation of rotor material [2].

The issue of rotor bow remains undetected until a rub occurs as most of the time operators do not look into phase amplitude plots during restart after short shut-down. There is no data base of such incident available to all compressor manufacturers / operators as the a) API 617 test procedure does not ask for hot restart b) most of the time soft inter-stage labyrinths are used.Baldassarre et al [1] have formulated a successfully implemented (hot restart sensitivity analysis) in design phase although the process is still its nascent stage (i.e. yet to be implemented by other compressor manufacturers).

\section{Worst case scenario}

The issue can lead to more profound effect due to high thermal gradient between a very short span of rotor when a back to back impeller configuration with cross over piping i.e. without any inter-stage cooling is used. With a bowed rotor having labyrinths with hard material, high radial vibration can lead to rotor -stator rub. The rotor can have deep scratch marks on sleeves and impeller hubs, partially wiped out division wall seal and blunted stainless steel labyrinth tooth.

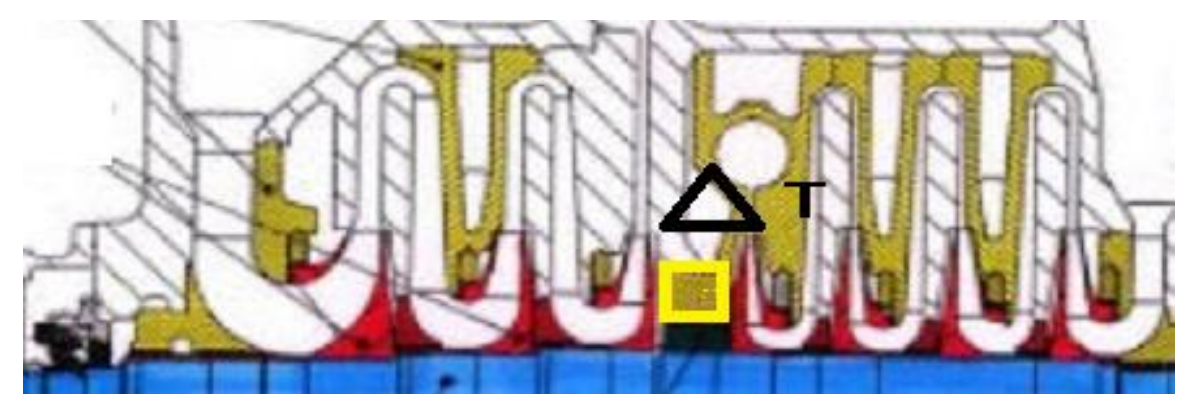

Fig2 - Back to back configuration with high thermal gradient $\Delta \mathrm{T}$ across center-wall

Subsequent to rub, material may be taken away by hard part of rotor (from balance drum and stationary labyrinth) and higher level of permanent unbalance was created. The compressor has to be dismantled to repair rotor and stator parts and compressor has to be subjected to mechanical run and performance tests. These activities may cause considerable delay in start-up of compressor.

\section{Dynamicsandseverity of rotor transient thermal bow}

The rotor phase angle with a bow can be considerably different than a rotor with residual unbalance. Thephase of mass eccentricity can be easily found out in MRT but it is quite difficult to predict the location of shaft bow (i.e. angle $\varphi$ between mass eccentricity vector $\delta$ sand shaft bow $\delta$ ). The total shaft deflection $\delta$ total is a vectorial sum of mass eccentricity vector $\delta$ s and shaft bow $\delta \mathrm{r}$.

It is possible to study and analyze the extent of bow and effect on rotor vibration amplitude by using phase amplitude plot from start up to resonance.

If the subject compressor is given multiple starts in short span of time (if driver permits), phase angle of unbalance and slow roll data changes at each start as the extent of bowing gets lessened

The force associated with a bent shaft is equal to the shaft stiffness times the bow radius. This is very different from mass unbalance case where force is associated with residual unbalanced mass, radius and speed squared.

As stated before in this paper that not all compressor rotor demonstrates the bow in explicit manner or cause serious threat to machinery integrity. The severity of bow depends on the magnitude of rotor bow compared to rotor residual unbalance (mass eccentricity) and phase angle between mass eccentricity $\delta$ s and shaft bow $\delta$ r. The below plots are shown to mathematically express the rotor behavior with a bow and unbalance (mass eccentricity). [3],[4] 

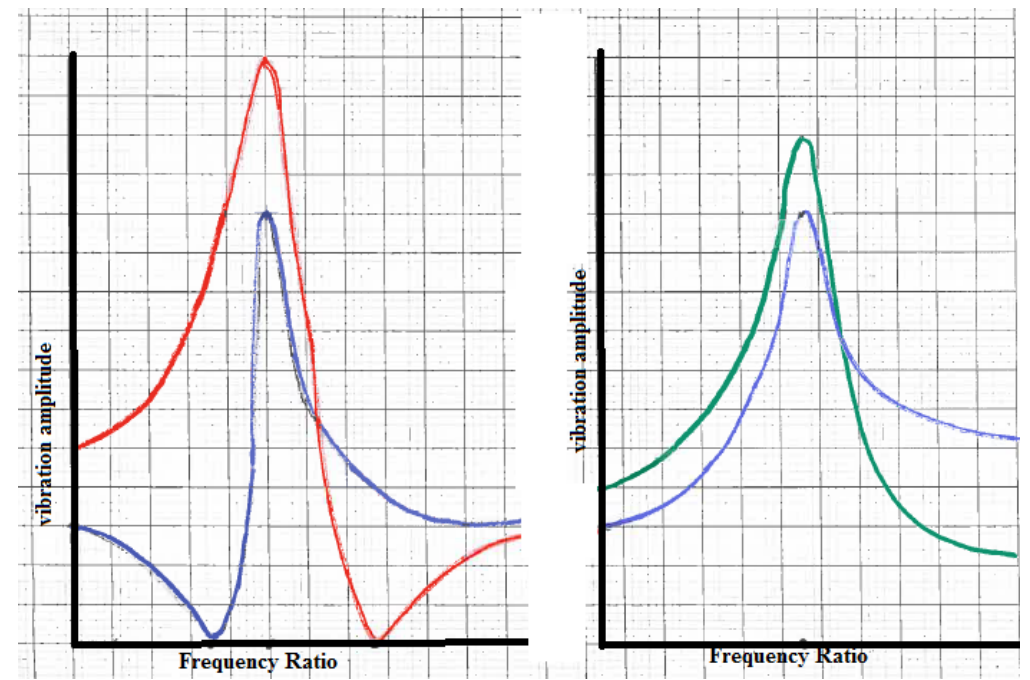

Fig 3 - Effect of Rotor bow and mass eccentricity on vibration amplitude

When the extent of thermal bow is less than mass eccentricity and they have phase difference of 180 degrees, the rotor vibration dips at value of $\omega / \omega n=0.707$ and goes to $\max$ at $\omega / \omega n=1$. This is shown in blue in plot at left side in fig 3. When the extent of bow is larger than mass eccentricity and they have phase difference of 180 degrees, the rotor vibration dips at value of $\omega / \omega n=1.414$.This is shown in red in plot at left side in fig 3 .

When the extent of thermal bow is less than mass eccentricity and they have phase difference of 90 degrees, the rotor vibration goes to $\max$ at $\omega / \omega n=1$.

When the extent of thermal bow equal to mass eccentricity and they have no phase difference, the rotor vibration goes to $\max$ at $\omega / \omega n=1$. The slow roll run out is directly proportional to extent of rotor bow. This is shown in right side of fig 3 . The severity of bow is classified as A if bow is less than eccentricity, bow is classified as B if bow is equal to eccentricity,bow is classified as $\mathrm{C}$ if bow is less than eccentricity.

The above can be used as logic solver to detect the severity (A, B, C)of bow shown below.

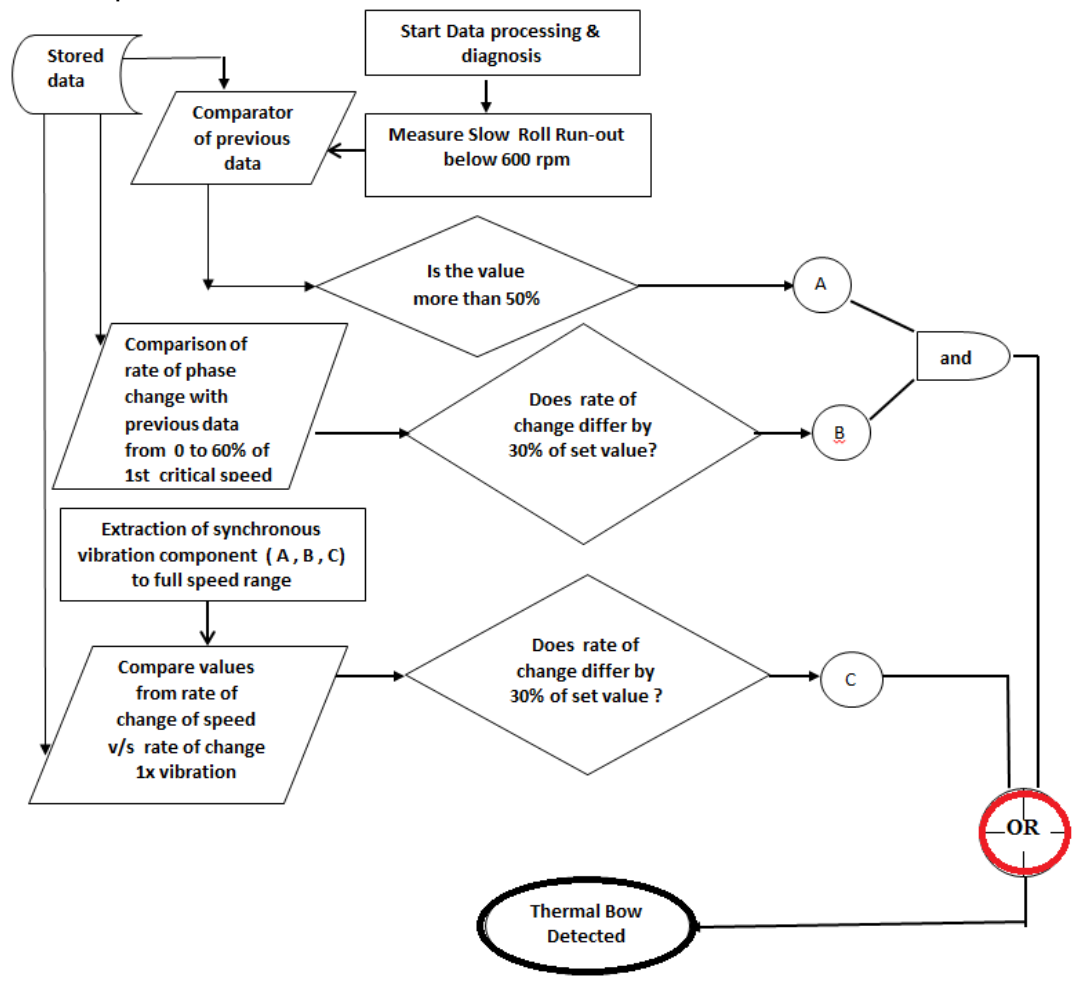

Fig 4- Logic Diagram to identify Rotor bow from mass eccentricity in 1x vibration

IV. Identification of Rotor bowat OEM works

Usually slow roll run out data is taken during startup and coast down in Bode plot. It is found that difference between slow roll data at start up and coast down after a prolonged run is considerable. 
Shaft displacement is frequently plotted in polar plot as phase and amplitude. In polar plot, unbalance vector and thermal vector can be identified; the change in vibration and phase angle within the polar plot from starting point to end of rigid body mode is termed as thermal vector.

A polar plot data along with Bode plot can be used in conjunction to detect residual thermal bow using phase reference during hot restart.

\section{Detecting susceptibility of rotor bow during tests at OEM works}

As an experiment to validate the phenomenon,one centrifugal compressor was spin tested as per API 617 edition 7 and peak amplitude and shaft slow roll data were takenat vendor works. The model of rotor response analysis to 4x API mid-span unbalance (with same properties of bearings) was kept as reference. After the mechanical run test (spintest), the subject compressor was put to full load full speed test keeping gas thermodynamic properties similar to contractual condition.

The comparison demonstrated the phenomena of rotor bow.

The response of rotor for $4 \mathrm{x}$ API i.e. $4 \mathrm{U}=6350 \mathrm{~W} / \mathrm{N}$ yielded $2.3 \mu$ mof eccentricity showing peak response as $14 \mu \mathrm{mpk}-\mathrm{pk}$ which was very close to unbalance test result.

After $4 \mathrm{hr}$. full load full speed test with a closed loop, the compressor was restarted after 25 minutes where the peak response at resonant condition was 72 microns which showed the shift of center of gravity by $12 \mu \mathrm{m}$ which confirmed the phenomena of rotor susceptibility to thermal bow. It can be seen that vibration was below $25 \mu \mathrm{m}$ (Red line limit)during steady state condition meeting the API 617 criteria.During the test alarm and trip settings were relaxed taking care of actual rotor to stator gap and measured amplification factor of rotor during unbalance response test.

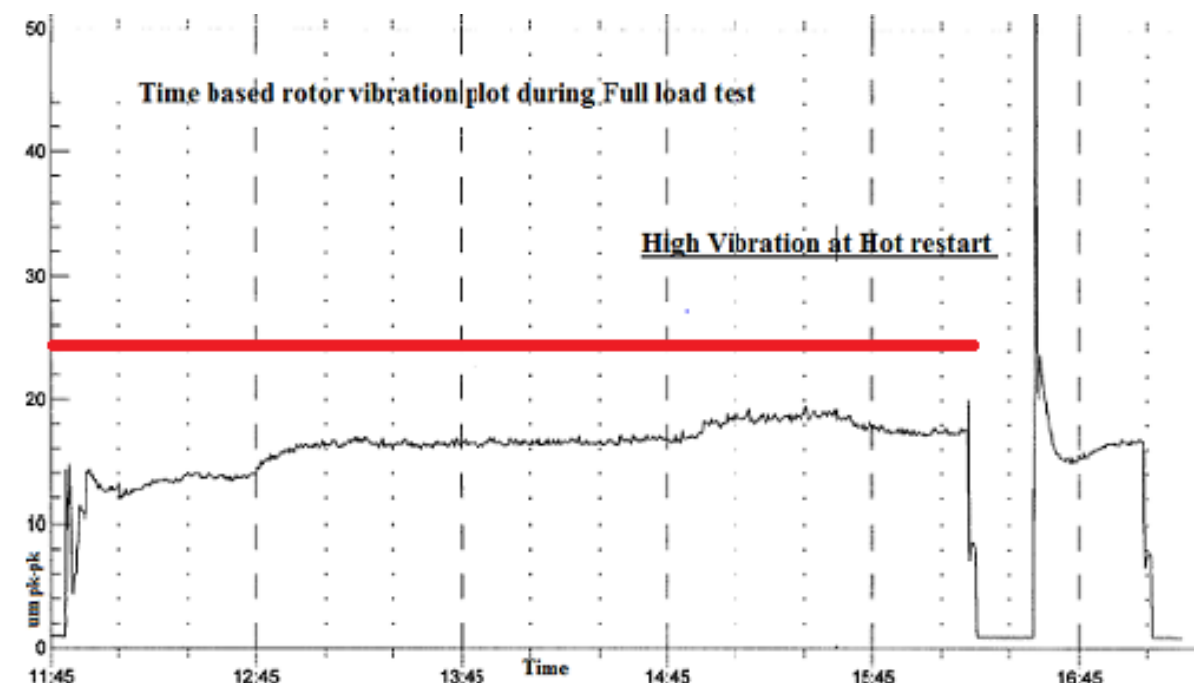

Fig 5a - High vibration after a short shutdown of 30 minutes and hot restart

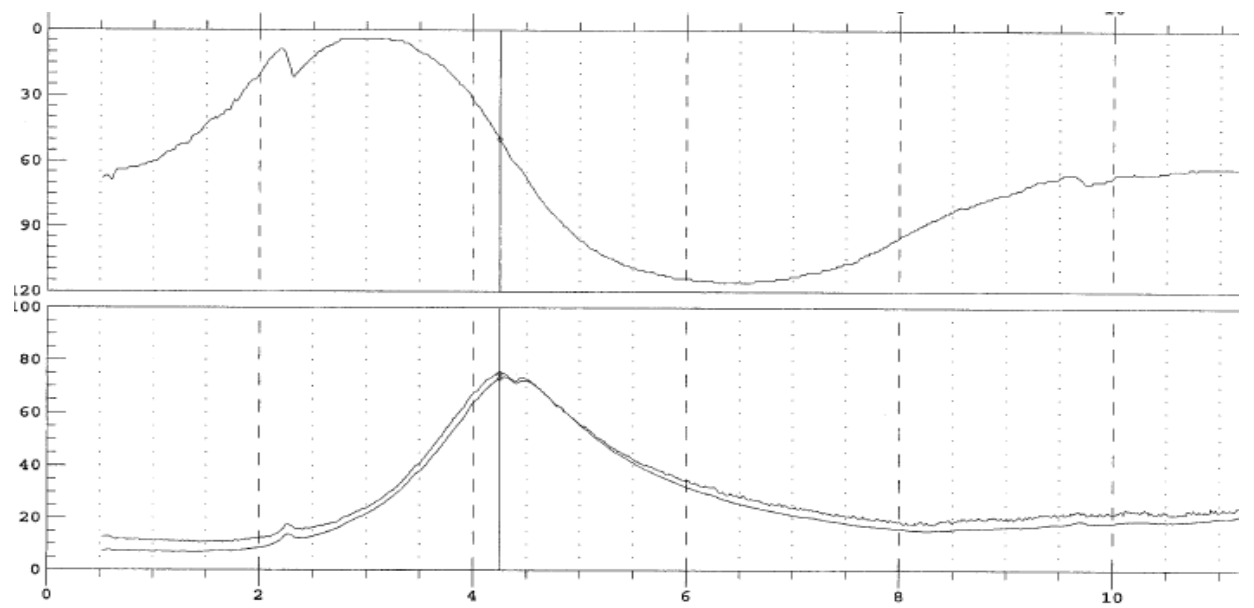

Fig 5b-Bode Plot showing high $1 \mathrm{X}$ vibration amplitude during hot restart

After spinning the rotor for 10 minutes well below the $1^{\text {st }}$ critical speed, the rotor vibration response was well belowthe high alarm value during restart. This means that,thermally induced bow shall disappear after 
spinning, unless the affected area has exceeded the yield limit of the material. This proves that transient eccentricity caused by differential cooling /heating of rotor is reversible.

It has been found that compressor having 5 pad LOP configuration do have high amplification factor on synchronous response to un balance in comparison to 4 pad LBP configuration as shown in table below .

\begin{tabular}{|c|c|c|c|c|c|c|c|c|c|c|}
\hline SN & \begin{tabular}{|c|} 
maximum \\
discharge \\
temperature \\
$\left({ }^{\circ} \mathrm{C}\right)$
\end{tabular} & RPM & $\begin{array}{c}\text { Rotor } \\
\text { Bearing } \\
\text { span } \\
(\mathrm{mm}) \\
\end{array}$ & $\begin{array}{l}\text { Rotor } \\
\text { weight } \\
\text { (kgs) }\end{array}$ & $\left|\begin{array}{c}\text { Type of } \\
\text { bearing } 4 \\
\text { Pad } / 5 \\
\text { pad }\end{array}\right|$ & $\begin{array}{l}\text { LOPI } \\
\text { LBP }\end{array}$ & $\begin{array}{l}\text { Pivot } \\
\text { offset }\end{array}$ & $\begin{array}{l}\text { 1st Critical } \\
\text { Speed }\end{array}$ & $\begin{array}{l}\text { interstage } \\
\text { labyrinth } \\
\text { material }\end{array}$ & $\begin{array}{c}\text { Synchronous } \\
\text { Amplification factor }\end{array}$ \\
\hline 1 & 177.8 & 10500 & 1690 & 641 & $5 \mathrm{Pad}$ & LOP & $60 \%$ & 4171 & $\begin{array}{l}\text { Aluminium } \\
\text { Alloy }\end{array}$ & 5.67 \\
\hline 2 & 169.1 & 11410 & 1625 & 523.83 & 4 Pad & LBP & Center & 5226 & $\begin{array}{c}\text { Stainless steed } \\
\text { (S31635) }\end{array}$ & 3.16 \\
\hline 3 & 174 & 9881 & 1733 & 661 & $4 \mathrm{Pad}$ & LOP & Center & 3815 & $\begin{array}{l}\text { Alurninium } \\
\text { Alloy }\end{array}$ & 4 \\
\hline 4 & \begin{tabular}{|l|} 
Sec-1: 142 \\
Sec-2: 133
\end{tabular} & 8028 & 2127 & 1002 & $5 \mathrm{Pad}$ & LOP & Certer & 3210 & $\begin{array}{l}\text { Alurninium } \\
\text { Aloy }\end{array}$ & 13.37 \\
\hline 5 & 135.9 & 11819 & 1382 & 387 & $5 \mathrm{Pad}$ & LOP & $60 \%$ & 6200 & $\begin{array}{l}\text { Aluminium } \\
\text { Aloy }\end{array}$ & 11.3 \\
\hline 6 & \begin{tabular}{|l|} 
Sec-1: 130 \\
Sec-2: 148
\end{tabular} & 11147 & 1658 & 627.2 & $5 \mathrm{Pad}$ & LOP & $60 \%$ & 4700 & ALSI 316 & 6 \\
\hline 7 & $\begin{array}{l}\text { Sec-1:96 } \\
\text { Sec-2: } 153\end{array}$ & 11975 & 1617 & 483.68 & 4 Pad & LBP & Certer & 4725 & $\begin{array}{c}\mathrm{X} 20 \mathrm{Cr} 13 \\
\text { (Martensitic } \\
\text { Stainless steel) }\end{array}$ & 25 \\
\hline 8 & 115 & 7316 & 2379 & 900 & $5 \mathrm{Pad}$ & LOP & $60 \%$ & 3113 & $\begin{array}{l}\text { Aluminium } \\
\text { Aloy }\end{array}$ & 11.8 \\
\hline 9 & 137 & 12554 & 1496 & 415 & 5 Pad & LOP & $60 \%$ & 4925 & $\begin{array}{l}\text { Aluminium } \\
\text { Aloy }\end{array}$ & 21.1 \\
\hline 10 & 117 & 11050 & 1283 & 304.3 & 5 Pad & LOP & $60 \%$ & 6176 & $\begin{array}{l}\text { Alurninium } \\
\text { Aloy }\end{array}$ & 6.09 \\
\hline
\end{tabular}

From row 6 and 7 from table, a direct comparison of amplification factors between two rotors with same bearing span and rotor weight can be made.

Effect of Honeycomb inter-stage / center-wall seals at hot restart during pressurized condition do not render much help to minimize $1 \mathrm{X}$ vibration till $1^{\text {st }}$ critical speed.

\section{Conclusion}

Based on above solutions, OEMmay have a combination of solutions unified into a standard solution for various machine train configurations.

The design phase should consider the requirement of modal sensitivity analysis of such rotors which can be prone to thermal transient vibrations during hot start- up of compressors

Finite Element analysis conducted by OEM can be very helpful to determine the loss of interference of shrink fit inter-stage shaft sleeves every time a bowed rotor passes through its first resonance speed. This can give an indication if the design allows a bowed rotor to pass through 1st critical speed.

\section{References}

[1]. Modeling of rotor bow during hot start of centrifugal compressor - L. Baldassarre\& M. Fontana 39th Turbo machinery symposium TAMU

[2]. Air Pressure Dependence of Natural-Convection Heat Transfer- MaysamSaidi, Reza HosseiniAbardeh- Proceedings of the World Congress on Engineering 2010 Vol II WCE 2010

[3]. Effect of residual shaft bow on unbalance response - Dr. John Nicholas, Dr. Edgar J. Gunter, Dr. P. Allaire.

[4]. Comparison of unbalance response of Jeffcott Rotor with shaft bow and shaft run out - Dr.R. DFlack, Dr. Edgar J. Gunter et al

[5] Start up issue with centrifugal compressor after a short shut down and mitigation to enhance reliability and availability -

M.Bhattacharya MFPT conference Dayton Ohio 2016

\section{Bibliography}

[1]. Practical Rotor-dynamics and Fluid Film Bearing Design by WenJeng Chen, 2015-Trafford publishing

[2]. Rotor dynamics, by Agnes Muszynska. CRC press 2005

[3]. Rotating Machinery Vibration: From Analysis to Troubleshooting by Maurice L. Adams CRC Press 2009. 